\title{
Stochastic Analysis of Bulk Production with Bulk and Catastrophic Sales System using Matrix Geometric Approach
}

\author{
Ramshankar.R \\ Independent Researcher \\ MS(ECE), University of Massachusetts \\ Amherst, MA, USA
}

\author{
Ramanarayanan. $R$ \\ Professor of Mathematics (Retired) \\ Veltech Group of Institutions, \\ Chennai India
}

\begin{abstract}
:
This paper studies two stochastic Models (A) and (B) with bulk production and bulk sales of products with catastrophe. The system has infinite storing capacity and the production and sales sizes are finite valued random variables with finite maximum production and sales sizes. When a catastrophe occurs the entire stocks are sold. Matrix partitioning method is used to study the models. In Model (A) the maximum production size is greater than the maximum sales size and the infinitesimal generator is partitioned as blocks of the maximum production size for analysis and in Model (B) the maximum production size is less than the maximum sales size and the latter is for partition. The stationary stock level probabilities, its expected values, its variances and probabilities of empty level are derived for the two models using the rate matrix iterated. Numerical examples are presented for illustration. Bulk arrival and bulk service queue becomes a special case of the model considered.
\end{abstract}

\section{General Terms}

Bulk Production, Bulk Sales, Catastrophe, Operating System, Bulk Queue and Markov Chain

\section{Keywords}

Block Sizes, Stationary Probability, Infinitesimal Generator and Matrix Geometric Approach.

\section{INTRODUCTION}

In this paper production and sales models are studied using matrix geometric methods. Aissani.A and Artalejo.J.R [1] and Ayyappan, Subramanian and Gopal Sekar [2] have analyzed retrial queueing system using matrix geometric methods. Bini, Latouche and Meini [3] have studied numerical methods for Markov chains and Chakravarthy and Neuts [4] have discussed in depth a multi-server queueing model. Gaver, Jacobs and Latouche [5] have treated birth and death models with random environment. Latouche and Ramaswami [6] have studied Analytic methods. For matrix geometric methods and models one may refer Neuts [7]. The models considered here are somewhat different from queueing theory models. Here random number of production and random number of sales of products are considered at a time whereas in queueing theory a fixed number of customers arrive or are served at any arrival or service epochs. Even in bulk service queueing model, when maximum service capacity is $b$, then fixed $b$ customers are cleared by a service when more than $b$ customers are waiting, Neuts and Nadarajan [8]. The models considered here being stocks, even when the maximum capacity of the selling system is $b$, only with some probability, the size $b$ may be cleared. In a similar way arrival sizes of customers are usually fixed in bulk arrival queueing systems but the models here treat bulk production of products subject to a probability law. Further in these models catastrophic sales can occur, clearing the entire stock of products produced. Such situations are seen often in software based industries where finished software projects waiting for marketing are sold when there is economic boom giving several fold profits. A similar situation also occurs when the hardware collapses or when the project team is slack and un-alert, all projects on hand may collapse. In industrial productions, bulk types are very common. Manufactured products arrive in several containers in marketing area and several bulk sizes of products are sold and at times the entire stock are cleared due to seasonal demands and clearance sale. Recently M/M/1 queueing systems with disaster has been studied by Noam Paz and Uri Yechali [9] but random arrival size or random service size have not been treated at any depth. Usually bulk arrival models have M/G/1 upper-Heisenberg block matrix structure with zeros below the first sub diagonal. The decomposition of a Toeplitz sub matrix of the infinitesimal generator is required to find the stationary probability vectors. Those vectors have no Matrix geometric structures as noted by William J. Stewart [10].

In this paper, two models (A) and (B) with random bulk production and random bulk sales are studied with infinite storage space for stocks. The block partitioning method is used to obtain matrix geometric results. In Model (A) the maximum production size $M$ is bigger than the maximum sales size $\mathrm{N}$. Its dual, the maximum sales size $\mathrm{N}$ is bigger than maximum production size $\mathrm{M}$ is considered in Model (B). In Queueing models, the state space of the system has the first co-ordinate indicating the number of customers in the system but here the customers (products) in the system are grouped and considered as members of $\mathrm{M}$ blocks (when $\mathrm{M}>\mathrm{N}$ ) or $\mathrm{N}$ blocks (when $\mathrm{N}>\mathrm{M}$ ) for finding the rate matrix. Using the maximum of the bulk sizes of production or sales for partitioning the infinitesimal generator is new approach in this area. Further catastrophic demands in this area has not been attempted so far. For systems with no catastrophe, the parameter used for the same may be considered as zero, $(\mathrm{a}=$ 0 ) and in this case, it becomes a random bulk queue in which arrivals occur in batches of random sizes and the services are provided in batches of random sizes. The stationary probability of the number products waiting for sales, the expectation and the variance of the number products waiting for sales and the probability of empty stock are derived. Numerical cases are presented to illustrate their applications.

\section{MODEL (A) MAXIMUM PRODUCTION SIZE GREATER THAN MAXIMUM SALES SIZE. $M$ > N.}

\subsection{Assumptions}

i) The inter-production time of products of bulk sizes has exponential distribution with parameter $\lambda$. At each production epoch $\chi$ products are produced with probability given by $\mathrm{P}$ 
$(\chi=\mathrm{j})=p_{j}$ for $1 \leq \mathrm{j} \leq \mathrm{M}$ and $\sum_{j=1}^{M} p_{j}=1$

ii) The time between consecutive epochs of bulk sizes of sales has exponential distribution with parameter $\mu$. At each sale epoch $\psi$ products are sold with probability $\mathrm{P}(\psi=\mathrm{j})=q_{j}$ for $1 \leq \mathrm{j} \leq \mathrm{N}$ when more than $\mathrm{N}$ products are available for sales where $\sum_{1}^{N} q_{j}=1$. When $\mathrm{n}$ products $(\mathrm{n}<\mathrm{N})$ are available, then $\mathrm{j}$ products are sold with probability $=p_{j}$, for $1 \leq \mathrm{i} \leq \mathrm{n}-1$ and $\mathrm{n}$ products are sold with probability $\sum_{n}^{N} p_{j}$.

iii) The inter occurrence time of catastrophe is exponential with parameter a. The stocks are all cleared when catastrophe occurs. The production of products begins afresh.

iv) The maximum production size $\mathrm{M}$ at a production epoch is greater than the maximum sales size $\mathrm{N}$ at a sale epoch.

\subsection{Analysis}

The state of the system of the continuous time Markov chain $\mathrm{X}(\mathrm{t})$ under consideration is presented as follows. $X(t)=\{(n, j)$ : for $0 \leq j \leq M-1$ and $0 \leq n<\infty\}$.

The chain is in the state $(n, j)$ when the stock level of products is $\mathrm{n} \mathrm{M}+\mathrm{j}$, for $0 \leq \mathrm{j} \leq \mathrm{M}-1$ and $0 \leq \mathrm{n}<\infty$. When the stock level in the system is $\mathrm{k}$ then $\mathrm{k}$ is identified with $(\mathrm{n}, \mathrm{j})$ where $\mathrm{k}$ on division by $M$ gives $n$ as the quotient and $j$ as the remainder. Let the survivor probability of products produced be $\mathrm{P}(\chi>\mathrm{i})=P_{i}=1-\sum_{j=1}^{i} \quad p_{j}$ for $\mathrm{i}=1,2 \ldots, \mathrm{M}-1$ and $P_{0}=1$. (2) Let the survivor probability of sales of products be $A_{1}=$

\begin{tabular}{|c|c|c|c|c|}
\hline$[-(\lambda+\mu+a)$ & $\lambda p_{1}$ & $\lambda p_{2}$ & $\ldots$ & $\lambda p_{M-N-2}$ \\
\hline$\mu q_{1}$ & $-(\lambda+\mu+a)$ & $\lambda p_{1}$ & $\cdots$ & $\lambda p_{M-N-3}$ \\
\hline$\mu q_{2}$ & $\mu q_{1}$ & $-(\lambda+\mu+a)$ & $\cdots$ & $\lambda p_{M-N-4}$ \\
\hline$\vdots$ & $\vdots$ & $\vdots$ & $\because$ & $\vdots$ \\
\hline$\mu q_{N}$ & $\mu q_{N-1}$ & $\mu q_{N-2}$ & $\cdots$ & $-(\lambda+\mu+a)$ \\
\hline 0 & $\mu q_{N}$ & $\mu q_{N-1}$ & $\cdots$ & $\mu q_{1}$ \\
\hline 0 & 0 & $\mu q_{N}$ & $\ldots$ & $\mu q_{2}$ \\
\hline$\vdots$ & $\vdots$ & $\vdots$ & $\ddots$ & $\vdots$ \\
\hline 0 & 0 & 0 & $\ldots$ & $\mu q_{N}$ \\
\hline 0 & 0 & 0 & $\ldots$ & 0 \\
\hline
\end{tabular}

$\mathrm{P}(\psi>\mathrm{i})=Q_{i}=1-\sum_{j=1}^{i} \quad q_{j}$ for $\mathrm{i}=1,2 \ldots, \mathrm{N}-1$ and $Q_{0}=1$. The chain $X(\mathrm{t})$ describing model has the infinitesimal generator $Q_{A}$ of infinite order which can be presented in block partitioned form given below.

$Q_{A}=\left[\begin{array}{cccccccc}B_{1} & A_{0} & 0 & 0 & . & . & . & \cdots \\ B_{2} & A_{1} & A_{0} & 0 & . & . & . & \cdots \\ B_{3} & A_{2} & A_{1} & A_{0} & 0 & . & . & \cdots \\ B_{3} & 0 & A_{2} & A_{1} & A_{0} & 0 & . & \ldots \\ B_{3} & 0 & 0 & A_{2} & A_{1} & A_{0} & 0 & \ldots \\ \vdots & \vdots & \vdots & \vdots & \ddots & \ddots & \ddots & \ddots\end{array}\right]$

In (4) the states of the matrices are listed lexicographically as $0, \underline{1}, 2,3, \ldots . n, \ldots$. Here $\underline{n}=((\mathrm{n}, 0),(\mathrm{n}, 1) \ldots \quad(\mathrm{n}, \mathrm{M}-1))$ for $\mathrm{n} \geq 0$. The matrices $B_{1}$ and $A_{1}$ have negative diagonal elements, they are of order $\mathrm{M}$ and their off diagonal elements are non- negative. The matrices $A_{0}, A_{2}, B_{2}$ and $B_{3}$ have nonnegative elements and are of order $\mathrm{M}$. They are below. $A_{0}=$

$\left[\begin{array}{ccccccc}\lambda p_{M} & 0 & 0 & \cdots & 0 & 0 & 0 \\ \lambda p_{M-1} & \lambda p_{M} & 0 & \cdots & 0 & 0 & 0 \\ \lambda p_{M-2} & \lambda p_{M-1} & \lambda p_{M} & \cdots & 0 & 0 & 0 \\ \lambda p_{M-3} & \lambda p_{M-2} & \lambda p_{M-1} & \ddots & 0 & 0 & 0 \\ \vdots & \vdots & \vdots & \ddots & \ddots & \vdots & \vdots \\ \lambda p_{3} & \lambda p_{4} & \lambda p_{5} & \cdots & \lambda p_{M} & 0 & 0 \\ \lambda p_{2} & \lambda p_{3} & \lambda p_{4} & \cdots & \lambda p_{M-1} & \lambda p_{M} & 0 \\ \lambda p_{1} & \lambda p_{2} & \lambda p_{3} & \cdots & \lambda p_{M-2} & \lambda p_{M-1} & \lambda p_{M}\end{array}\right]$

$$
\left.\begin{array}{ccccc}
\lambda p_{M-N-1} & \lambda p_{M-N} & \cdots & \lambda p_{M-2} & \lambda p_{M-1} \\
\lambda p_{M-N-2} & \lambda p_{M-N-1} & \cdots & \lambda p_{M-3} & \lambda p_{M-2} \\
\lambda p_{M-N-3} & \lambda p_{M-N-2} & \cdots & \lambda p_{M-4} & \lambda p_{M-3} \\
\vdots & \vdots & \ddots & \vdots & \vdots \\
\lambda p_{1} & \lambda p_{2} & \cdots & \lambda p_{M-N-2} & \lambda p_{M-N-1} \\
-(\lambda+\mu+a) & \lambda p_{1} & \cdots & \lambda p_{M-N-3} & \lambda p_{M-N-2} \\
\mu q_{1} & -(\lambda+\mu+a) & \cdots & \lambda p_{M-N-4} & \lambda p_{M-N-3} \\
\vdots & \vdots & \ddots & \vdots & \vdots \\
\mu q_{N-1} & \mu q_{N-2} & \cdots & -(\lambda+\mu+a) & \lambda p_{1} \\
\mu q_{N} & \mu q_{N-1} & \cdots & \mu q_{1} & -(\lambda+\mu+a)
\end{array}\right]
$$

$B_{1}=$

$\left[\begin{array}{cccccccccc}-\lambda & \lambda p_{1} & \lambda p_{2} & \cdots & \lambda p_{M-N-2} & \lambda p_{M-N-1} & \lambda p_{M-N} & \cdots & \lambda p_{M-2} & \lambda p_{M-1} \\ \mu+a & -(\lambda+\mu+a) & \lambda p_{1} & \cdots & \lambda p_{M-N-3} & \lambda p_{M-N-2} & \lambda p_{M-N-1} & \cdots & \lambda p_{M-3} & \lambda p_{M-2} \\ \mu Q_{1}+a & \mu q_{1} & -(\lambda+\mu+a) & \cdots & \lambda p_{M-N-4} & \lambda p_{M-N-3} & \lambda p_{M-N-2} & \cdots & \lambda p_{M-4} & \lambda p_{M-3} \\ \vdots & \vdots & \vdots & \ddots & \vdots & \vdots & \vdots & \ddots & \vdots & \vdots \\ \mu Q_{N-1}+a & \mu q_{N-1} & \mu q_{N-2} & \cdots & -(\lambda+\mu+a) & \lambda p_{1} & \lambda p_{2} & \cdots & \lambda p_{M-N-2} & \lambda p_{M-N-1} \\ 0 & \mu q_{N} & \mu q_{N-1} & \cdots & \mu q_{1} & -(\lambda+\mu+a) & \lambda p_{1} & \cdots & \lambda p_{M-N-3} & \lambda p_{M-N-2} \\ 0 & 0 & \mu q_{N} & \cdots & \mu q_{2} & \mu q_{1} & -(\lambda+\mu+a) & \cdots & \lambda p_{M-N-4} & \lambda p_{M-N-3} \\ \vdots & \vdots & \vdots & \ddots & \vdots & \vdots & \vdots & \ddots & \vdots & \vdots \\ 0 & 0 & 0 & \cdots & \mu q_{N} & \mu q_{N-1} & \mu q_{N-2} & \cdots & -(\lambda+\mu+a) & \lambda p_{1} \\ 0 & 0 & 0 & \cdots & 0 & \mu q_{N} & \mu q_{N-1} & \cdots & \mu q_{1} & -(\lambda+\mu+a)\end{array}\right]$

$B_{2}=A_{2}+B_{3}$ where $\quad B_{3}=\left[\begin{array}{cccc}a & 0 & \cdots & 0 \\ a & 0 & \cdots & 0 \\ a & 0 & \cdots & 0 \\ \vdots & \vdots & \vdots & \vdots \\ a & 0 & \cdots & 0\end{array}\right](8)$ 


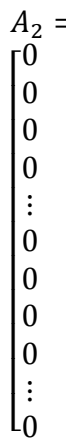

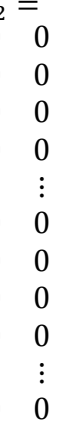

$\begin{array}{cc}\cdots & 0 \\ \cdots & 0 \\ \cdots & 0 \\ \cdots & 0 \\ \vdots & \vdots \\ \cdots & 0 \\ \cdots & 0 \\ \cdots & 0 \\ \cdots & 0 \\ \vdots & \vdots \\ \cdots & 0\end{array}$

$\mu q_{N-2}$
$\mu q_{N-1}$
$\mu q_{N}$
0
$\vdots$
0
0
0
0
$\vdots$
0

$\begin{array}{ll}\cdots & \mu q_{3} \\ \cdots & \mu q_{4} \\ \cdots & \mu q_{5} \\ \ddots & \mu q_{6} \\ \ddots & \\ \cdots & \mu q \\ \cdots & \\ . . & 0 \\ . . & 0 \\ \vdots & \\ \cdots & \end{array}$ (iii) The expected stock level can be calculated as follows. Since $\pi(\mathrm{n}, \mathrm{j})=\mathrm{P}[$ stock level $=\mathrm{Mn}+\mathrm{j}]$, for $\mathrm{n} \geq 0$, and $1 \leq \mathrm{j} \leq$ $\mathrm{M}-1$, we find $\mathrm{E}(\mathrm{S})=\sum_{n=0}^{\infty} \sum_{j=0}^{M-1}(M n+j) \pi(n, j)$

$=\sum_{n=0}^{\infty} \pi_{n} .(\mathrm{M} \mathrm{n}, \mathrm{Mn}+1, \mathrm{Mn}+2, \ldots, \mathrm{Mn}+\mathrm{M}-1)$

$=\mathrm{M} \sum_{n=0}^{\infty} n \pi_{n} e+\pi_{0}(I-R)^{-1} .(0,1,2,3, \ldots, M-1)$.

This gives, $\quad \mathrm{E}(\mathrm{S})=M \pi_{0}(I-R)^{-2} R e$

$+\pi_{0}(I-R)^{-1} \cdot(0,1,2, \ldots, M-1)$

(iv) Variance of the stock level can be derived easily as follows. Now $\mathrm{E}\left(S^{2}\right)=\sum_{n=0}^{\infty} \sum_{J=0}^{M-1}[M n+j]^{2} \pi(n, j)$

$=M^{2}\left[\sum_{n=1}^{\infty} n(n-1) \pi_{n} e+\sum_{n=0}^{\infty} n \pi_{n} e\right]$

$+\sum_{n=0}^{\infty} \pi_{n} \cdot\left[0,1^{2}, 2^{2}, 3^{2}, \ldots,(M-1)^{2}\right]$

$+2 \mathrm{M} \sum_{n=0}^{\infty} n \pi_{n} \cdot(0,1,2, \ldots \ldots, M-1)$. On simplification

$\mathrm{E}\left(S^{2}\right)=M^{2}\left[\pi_{0}(I-R)^{-3} 2 R^{2} e+\pi_{0}(I-R)^{-2} R e\right]$

$+\pi_{0}(I-R)^{-1} \cdot\left(0,1,2^{2}, 3^{2}, \ldots .,(M-1)^{2}\right)$

$+2 \mathrm{M}\left[\pi_{0}(I-R)^{-2} R .[0,1,2,3, \ldots \ldots,(M-1)]\right.$

production and sales including catastrophe is $\mathrm{Q}^{\prime}=A_{0}+A_{1}+A_{2}+B_{3}$

When $\mathrm{w}$ is its probability vector then $\mathrm{w} \mathrm{Q}^{\prime}=0$ and $\mathrm{w} \mathrm{e}=1$. Neuts [7], gives the stability condition as, $\mathrm{w}_{0} e<w A_{2} e$ where $\mathrm{W}=\left(w_{1}, w_{2}, \ldots . w_{M}\right)$ which reduces to $\lambda \sum_{i=1}^{M} w_{i} P_{M-i}<\mu \sum_{i=1}^{N} w_{i} Q_{i-1}$

where $P_{i}$ and $Q_{j}$ are given by (2) and (3) for $1 \leq \mathrm{i} \leq \mathrm{M}-1$ and for $1 \leq \mathrm{j} \leq \mathrm{N}-1$ with $P_{0}=Q_{0}=1$. When the inequality (11) is satisfied, the stationary distribution exists, Neuts [7]. Let $\pi(n, j)$, for $0 \leq j \leq M-1$ and $0 \leq n<\infty$ be the stationary probability of the states in (1) and $\pi_{n}$ be the vector of type $1 \times$ M with, $\pi_{n}=(\pi(\mathrm{n}, 0), \pi(\mathrm{n}, 1), \ldots \pi(\mathrm{n}, \mathrm{M}-1)), \mathrm{n} \geq 0$. (12) The stationary probability vector $\pi=\left(\pi_{0}, \pi_{1}, \pi_{3} \ldots,\right)$ satisfies $\pi Q_{A}=0$, and $\pi \mathrm{e}=1$.

From (13), $\pi_{0} B_{1}+\pi_{1} B_{2}+\sum_{2}^{\infty} \pi_{i} B_{3}=0$.

$\pi_{n-1} A_{0}+\pi_{n} A_{1}+\pi_{n+1} A_{2}=0$, for $\mathrm{n} \geq 1$.

Introducing the rate matrix $\mathrm{R}$ as the minimal non-negative
solution of the non-linear matrix equation $A_{0}+\mathrm{R} A_{1}+R^{2} A_{2}=0$

it can be proved (Neuts [7]) that $\pi_{n}$ satisfies the following. $\pi_{n}=\pi_{0} R^{n} \quad$ for $\mathrm{n} \geq 1$.

Using (17) and (14), it can be seen that $\pi_{0}$ satisfies $\pi_{0}\left[B_{1}+R A_{2}+(I-R)^{-1} R B_{3}\right]=0$.

It can be seen that the multiplier matrix of $\pi_{0}$ is a generator. Taking $(I-R)^{-1}$ as a common factor it can be seen that $(I-R)^{-1}\left[(I-R)\left(B_{1}+R A_{2}\right)+R B_{3}\right] e$

$=(I-R)^{-1}\left[B_{1} e-R B_{1} e+R A_{2} e+R B_{3} e-R^{2} A_{2} e\right]$

Using the row sum is zero in $Q_{A}$ and using (16), this is $=(I-R)^{-1}\left[-A_{0} e-R B_{1} e+R A_{2} e+R B_{3} e+A_{0} e+R A_{1} e\right]$ $=(I-R)^{-1} R\left[-B_{1} e+B_{3} e+A_{2} e+A_{1} e\right] \quad=0$. So using (18) the vector $\pi_{0}$ can be calculated up to multiplicative constant level. The normalizer in equation (13) gives, $\pi_{0}(I-R)^{-1} e=1$.

Replacing the first column of the matrix multiplier of $\pi_{0}$ in equation (18) by the column vector multiplier of $\pi_{0}$ in (19), a matrix which is invertible may be obtained. The first row of the inverse of that same matrix is $\pi_{0}$.This gives along with (17) all stationary probabilities of the system. The matrix $\mathrm{R}$ is computed by successive substitutions in the recur relation $R(0)=0 ; R(n+1)=-A_{0} A_{1}^{-1}-R^{2}(n) A_{2} A_{1}^{-1}, \mathrm{n} \geq 0$. (20). The iteration may be terminated to get a solution of $\mathrm{R}$ at a norm level where $\|R(n+1)-R(n)\|<\varepsilon$.

\subsection{Performance Measures}

(i) The probability of the stock level is $n$ can be seen as follows. Let $\mathrm{k} \geq 0$ and $\mathrm{j}$ for $0 \leq \mathrm{j} \leq \mathrm{M}$ be non-negative integers such that $\mathrm{n}=\mathrm{k} \mathrm{M}+\mathrm{j}$. Then using (17) and (12) it is noted that $\mathrm{P}($ Stock level $=\mathrm{n})=\pi(k, j)$, where $\mathrm{n}=\mathrm{k} \mathrm{M}+\mathrm{j}$.

(ii) The probability that the stock level is zero $=$

$\mathrm{P}($ Stock level $=0)=\pi(1,0)$.
$\operatorname{Var}(\mathrm{S})=\mathrm{E}\left(S^{2}\right)-[E(S)]^{2}$ may be written.

\subsection{Special Case: $M / M / 1$ Bulk Arrival and Bulk Service Queue with Maximum Arrival Size M > Maximum Service Size N}

It can be seen that when the catastrophic parameter $\mathrm{a}=0$ the model reduces to a bulk arrival and bulk service queueing system with both arrival and service batches are of random sizes. The matrices given above $A_{i}$ and $B_{j}$ for $\mathrm{i}=0,1,2$ and $\mathrm{j}$ $=1,2,3$ and $Q_{A}$ and $Q^{\prime}$ are all same here with a $=0$ where ever a appears. Since bulk arrival processes has M/G/1 queueing structure so far no attempt has been made for identifying the fine matrix geometric structure. For $\mathrm{M}>\mathrm{N}$ it is interesting to find the Queueing model has Matrix geometric solution when stability condition given in [7], w $A_{0} e<$ $w A_{2} e$ is satisfied where $\mathrm{w} \mathrm{Q}^{\prime}=0$ and $\mathrm{w} \mathrm{e}=1$ which is given in (11) $\quad \lambda \sum_{i=1}^{M} w_{i} P_{M-i}<\mu \sum_{i=1}^{N} w_{i} Q_{i-1}$. It is very interesting to note that the matrix $\mathrm{Q}^{\prime}=A_{0}+A_{1}+A_{2}$ is a circulant matrix which is a special kind of Toeplitz matrix where each row vector is rotated one element to the right relative to the preceding row vector. The matrix Q'is of order $M$ with row sum $=0$ and because of the circulant nature, the column sum $=$ row sum and are both $=0$ which gives the left eigen vector has 1 as its all coordinates for eigen value $=0$. Using the normalizing condition w.e $=1$, it can be seen that

$w=\left(\frac{1}{M}, \frac{1}{M}, \frac{1}{M}, \ldots, \frac{1}{M}\right)$.

Since $\sum_{i=1}^{M} P_{M-i}=\mathrm{E}(\chi),(=$ expected bulk arrival size $)$ and $\sum_{i=1}^{N} Q_{i-1}=\mathrm{E}(\psi),(=$ expected bulk service size $)$, the stability condition for the bulk size queue with maximum arrival size greater than maximum service size is, $\lambda \mathrm{E}(\chi)<\mu \mathrm{E}(\psi)$. (24) Section 2.4 presents all performance measures including expected length and variance for this special case also.

\section{MODEL (B) MAXIMUM PRODUCTION SIZE LESS THAN MAXIMUM SALES SIZE. $M<N$}

In this Model (B) the dual case of Model (A) is treated. (When the maximum production and sales sizes are equal both models are applicable and one can use any one of them.) The assumption (iv) of Model (A) alone is modified. 3.1Assumptions

(iv)The maximum production size $\mathrm{M}$ at a production epoch is less than the maximum sales size $\mathrm{N}$ at a sale epoch. 


\subsection{Analysis}

Since the model is dual, the analysis is same as that of Model (A). The differences are noted below. The state space of the chain as follows in a similar way.

$X(t)=\{(n, j)$ : for $0 \leq j \leq N-1$ and $0 \leq n<\infty\}$.

The chain is in the state $(n, j)$ when the stock level of products is, $\mathrm{nN}+\mathrm{j}$, for $0 \leq \mathrm{j} \leq \mathrm{N}-1$ and $0 \leq \mathrm{n}<\infty$. When the stock level in the system is $k$ then $k$ is identified with $(n, j)$ where $k$ on division by $\mathrm{N}$ gives $\mathrm{n}$ as the quotient and $\mathrm{j}$ as the remainder. The infinitesimal generator $Q_{B}$ of the model has the same block partitioned structure given in (4) for Model (A) but the inner matrices are of different orders and elements. They are listed below.

$Q_{B}=\left[\begin{array}{cccccccc}B^{\prime}{ }_{1} & A^{\prime}{ }_{0} & 0 & 0 & . & . & . & \cdots \\ B^{\prime}{ }_{2} & A^{\prime}{ }_{1} & A^{\prime}{ }_{0} & 0 & . & . & . & \cdots \\ B^{\prime}{ }_{3} & A^{\prime}{ }_{2} & A^{\prime}{ }_{1} & A^{\prime}{ }_{0} & 0 & . & . & \cdots \\ B^{\prime}{ }_{3} & 0 & A^{\prime}{ }_{2} & A^{\prime}{ }_{1} & A^{\prime}{ }_{0} & 0 & . & \cdots \\ B^{\prime}{ }_{3} & 0 & 0 & A^{\prime}{ }_{2} & A^{\prime}{ }_{1} & A^{\prime}{ }_{0} & 0 & \cdots \\ \vdots & \vdots & \vdots & \vdots & \ddots & \ddots & \ddots & \ddots\end{array}\right]$

In (26) the states of the matrices are listed lexicographically as $\underline{0}, \underline{1}, \underline{2}, \underline{3}, \ldots . \underline{n}, \ldots$. Here the state vector is given as follows. $\underline{n}=((\mathrm{n}, 0),(\mathrm{n}, 1),(\mathrm{n}, 2), \ldots \ldots \ldots(\mathrm{n}, \mathrm{N}-1))$, for $0 \leq \mathrm{n}<\infty$. $\bar{A}_{1}^{\prime}=$

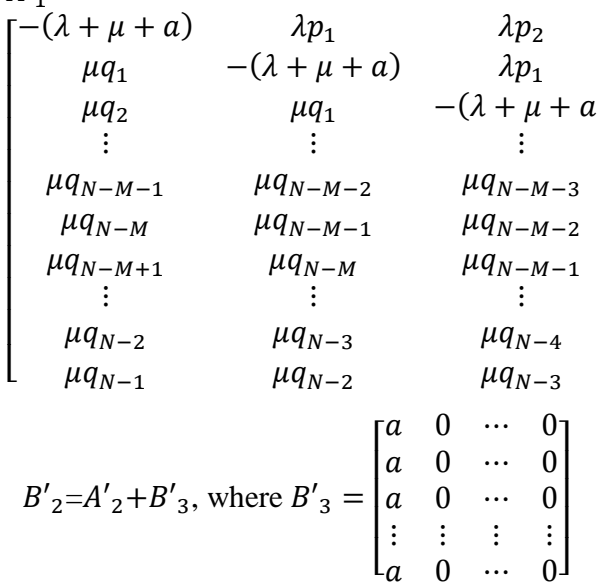

The basic generator which is concerned with only the production and sales including catastrophe is

$Q_{B}^{\prime}=A_{0}^{\prime}+A_{1}^{\prime}+A_{2}^{\prime}+B_{3}^{\prime} \quad$ (31) exists, Neuts [7]

The matrices, $B_{1}^{\prime}, B_{2}^{\prime}, B_{3}^{\prime}, A^{\prime}{ }_{0}, A_{1}^{\prime}$ and $A^{\prime}{ }_{2}$ are all of order $\mathrm{N}$. The matrices, $B^{\prime}{ }_{1}$ and $A^{\prime}{ }_{1}$ have negative diagonal elements and their off diagonal elements are non- negative. The matrices $A_{0}^{\prime}, A^{\prime}{ }_{2}, B^{\prime}{ }_{2}$ and $B^{\prime}{ }_{3}$ have nonnegative elements. They are all given below.

$$
\begin{aligned}
A^{\prime} & =\left[\begin{array}{cccccccc}
0 & 0 & \cdots & 0 & 0 & 0 & \cdots & 0 \\
\vdots & \vdots & \cdots & \vdots & \vdots & \vdots & \vdots & \vdots \\
0 & 0 & \cdots & 0 & 0 & 0 & \cdots & 0 \\
\lambda p_{M} & 0 & \cdots & 0 & 0 & 0 & \cdots & 0 \\
\lambda p_{M-1} & \lambda p_{M} & \cdots & 0 & 0 & 0 & \cdots & 0 \\
\vdots & \vdots & \ddots & \vdots & \vdots & \vdots & \vdots & \vdots \\
\lambda p_{2} & \lambda p_{3} & \cdots & \lambda p_{M} & 0 & 0 & \cdots & 0 \\
\lambda p_{1} & \lambda p_{2} & \cdots & \lambda p_{M-1} & \lambda p_{M} & 0 & \cdots & 0
\end{array}\right](27) \\
A^{\prime}{ }_{2} & =\left[\begin{array}{ccccc}
\mu q_{N} & \mu q_{N-1} & \cdots & \mu q_{2} & \mu q_{1} \\
0 & \mu q_{N} & \cdots & \mu q_{3} & \mu q_{2} \\
0 & 0 & \cdots & \mu q_{4} & \mu q_{3} \\
0 & 0 & \ddots & \mu q_{5} & \mu q_{4} \\
\vdots & \vdots & \ddots & \vdots & \vdots \\
0 & 0 & \cdots & \mu q_{N-1} & \mu q_{N-2} \\
0 & 0 & \cdots & \mu q_{N} & \mu q_{N-1} \\
0 & 0 & \cdots & 0 & \mu q_{N}
\end{array}\right]
\end{aligned}
$$

$B^{\prime}{ }_{1}=$
$\left[\begin{array}{ccccc}-\lambda & \lambda p_{1} & \lambda p_{2} & \cdots & \lambda p_{M} \\ a+\mu & -(\lambda+\mu+a) & \lambda p_{1} & \cdots & \lambda p_{M-1} \\ a+\mu Q_{1} & \mu q_{1} & -(\lambda+\mu+a) & \cdots & \lambda p_{M-2} \\ \vdots & \vdots & \vdots & \ddots & \vdots \\ a+\mu Q_{N-M-2} & \mu q_{N-M-2} & \mu q_{N-M-3} & \cdots & -(\lambda+\mu+a) \\ a+\mu Q_{N-M-1} & \mu q_{N-M-1} & \mu q_{N-M-2} & \cdots & \mu q_{1} \\ a+\mu Q_{N-M} & \mu q_{N-M} & \mu q_{N-M-1} & \cdots & \mu q_{2} \\ \vdots & \vdots & \vdots & \ddots & \vdots \\ a+\mu Q_{N-3} & \mu q_{N-3} & \mu q_{N-4} & \cdots & \mu q_{N-M-2} \\ a+\mu Q_{N-2} & \mu q_{N-2} & \mu q_{N-3} & \cdots & \mu q_{N-M-1}\end{array}\right.$

Let $\pi(n, j)$, for $0 \leq \mathrm{j} \leq \mathrm{N}-1$ and $0 \leq \mathrm{n}<\infty$ be the stationary probability of the states listed in (25) and $\pi_{n}$ for $0 \leq \mathrm{n}<\infty$ be the vector of type $1 \times \mathrm{N}$ given by respectively, $\pi_{n}=(\pi(\mathrm{n}, 0), \pi(\mathrm{n}, 1), \ldots \ldots, \pi(\mathrm{n}, \mathrm{N}-2), \pi(\mathrm{n}, \mathrm{N}-1))$, for $\mathrm{n} \geq 0$. (34) The stationary probability vector $\pi=\left(\pi_{0}, \pi_{1}, \pi_{3}, \ldots\right)$ satisfies $\pi Q_{B}=0$, and $\pi \mathrm{e}=1$.

$\left.\begin{array}{ccccc}0 & 0 & \cdots & 0 & 0 \\ \lambda p_{M} & 0 & \cdots & 0 & 0 \\ \lambda p_{M-1} & \lambda p_{M} & \cdots & 0 & 0 \\ \vdots & \vdots & \ddots & \vdots & \vdots \\ \lambda p_{1} & \lambda p_{2} & \cdots & \lambda p_{M-1} & \lambda p_{M} \\ -(\lambda+\mu+a) & \lambda p_{1} & \cdots & \lambda p_{M-2} & \lambda p_{M-1} \\ \mu q_{1} & -(\lambda+\mu+a) & \cdots & \lambda p_{M-3} & \lambda p_{M-2} \\ \vdots & \vdots & \ddots & \vdots & \vdots \\ \mu q_{N-M-3} & \mu q_{N-M-2} & \cdots & -(\lambda+\mu+a) & \lambda p_{1} \\ \mu q_{N-M-2} & \mu q_{N-M-1} & \cdots & \mu q_{1} & -(\lambda+\mu+a)\end{array}\right]$

(33)

Following the arguments given for Model (A), one can find the stationary probability vector for Model (B) also in matrix geometric form. Performance measures including expectation of products waiting for sales and its variance for Model (B) have the same form as given in Model (A) except (maximum production size) $\mathrm{M}$ is replaced by (maximum sales size) $\mathrm{N}$. 


\subsection{Special Case: Bulk Arrival and Bulk} Service Queue with $\mathbf{M}<\mathbf{N}$ It can be seen that when the catastrophic parameter $\mathrm{a}=0$ the model reduces to a bulk arrival and bulk service queueing system with both arrival and service batches of random sizes. Results already derived for the case when $\mathrm{M}>\mathrm{N}$. are also applicable. For $\mathrm{M}<\mathrm{N}$, the matrix $Q_{B}^{\prime}{ }_{B}=A^{\prime}{ }_{0}+A^{\prime}{ }_{1}+A^{\prime}{ }_{2}$ is again circulant matrix with both row sum $=$ column sum $=0$. This gives $w^{\prime}=\left(\frac{1}{N}, \frac{1}{N}, \frac{1}{N}, \ldots, \frac{1}{N}\right)$. Since $\sum_{i=1}^{M} P_{M-i}=\mathrm{E}(\chi),(=$ expected arrival size $)$ and $\sum_{i=1}^{N} Q_{i-1}=\mathrm{E}(\psi),(=$ expected service size $)$, the stability condition for the bulk size queue with maximum arrival size < maximum service size is given by $\lambda \mathrm{E}(\chi)<\mu \mathrm{E}(\psi)$.(37) For all performance measures including expected length and variance results obtained in section 2.4 may be used.

\section{NUMERICAL ILLUSTRATIONS}

Three numerical examples each for the two models are presented below. The production parameter is fixed as $\lambda=3$. The parameter $\mu$ of the sales time varies as $\mu=6,7,8$ respectively in the two Models (A) and (B). The assigned maximum sizes $\mathrm{M}$ and $\mathrm{N}$ and values of the probabilities for the sizes of production and sales for Models (A) and (B) are given in table 1 . The various probabilities $\mathrm{P}(\mathrm{S}=0), \mathrm{P}(\mathrm{S}=1)$, $\mathrm{P}(\mathrm{S}=2), \mathrm{P}(\mathrm{S}=3)$ and $\mathrm{P}(\mathrm{S}>20)$ of the stock level $\mathrm{S}=0,1,2,3$ and more than 20 are calculated. The probabilities $\pi_{0} e, \pi_{1} e$ and $\pi_{2} e$ of the stock level $\mathrm{S}$ in the levels $0 \leq \mathrm{S} \leq 6$, $7 \leq \mathrm{S} \leq 13$ and $14 \leq \mathrm{S} \leq 20$, expected stock level, the variance of the stock level are obtained using the rate matrix $R$ employing the numerical iteration method presented in (20). The iteration is performed up to E-05 level for $\varepsilon$. The matrix R converges faster in Model (B) since the matrix $A_{0}$ has more zero rows in Model (B) which makes the matrix R has same property. In the two models when $\mathrm{d}$ increases, sales rate increases whereas $E(S)$ and Variance of $S$ decrease. All probabilities show significant variations and $P(S>20)$ is very much small. Figures 1 and 4 present probabilities of stock level at $0,1,2$ and 3 for different sales rates and figures 2 and 4 present sum of probabilities of first and second blocks of 7 to 13 and 14 to 20 stocks and $P(S>20)$. In figures 3 and 6 probabilities of block zero, 0 to 6 stocks, $E(S)$ and sales rate are presented. In the two models when sales rate increases, $\mathrm{E}(\mathrm{S})$ decreases. As the parameter $\lambda$ of the production process increases, in Model (A) the effective production rate w $A_{0} e$ increases. This together with the presence of the catastrophic rate causes significant change in the stationary probability vector of the system generator matrix $Q_{A}$ causing the increase in the effective sales rates, $w A_{2} e$ as presented in inequality (11). The situation is same also in Model (B) where the generator is $Q_{B}^{\prime}$. When the catastrophic rate $=0$, the basic system generator matrices in the two models become circulant as seen in the two special cases of bulk queues where the stationary probability vector of the generator has equal values for all its components, equal to inverse of the maximum of $\mathrm{M}$ or $\mathrm{N}$, as the case may be, the effective service rate remains $\mu$ $\mathrm{E}(\psi)$ as seen in (24).

Table 1. Values of $M, N$ and probabilities of production and sales.

\begin{tabular}{|l|l|l|l|l|l|l|l|l|l|l|l|l|l|l|l|l|}
\hline Model & $\mathbf{M}$ & $\mathbf{N}$ & $\boldsymbol{p}_{\mathbf{1}}$ & $\boldsymbol{p}_{\mathbf{2}}$ & $\boldsymbol{p}_{\mathbf{3}}$ & $\boldsymbol{p}_{\mathbf{4}}$ & $\boldsymbol{p}_{\mathbf{5}}$ & $\boldsymbol{p}_{\mathbf{6}}$ & $\boldsymbol{p}_{\mathbf{7}}$ & $\boldsymbol{q}_{\mathbf{1}}$ & $\boldsymbol{q}_{\mathbf{2}}$ & $\boldsymbol{q}_{\mathbf{3}}$ & $\boldsymbol{q}_{\mathbf{4}}$ & $\boldsymbol{q}_{\mathbf{5}}$ & $\boldsymbol{q}_{\mathbf{6}}$ & $\boldsymbol{q}_{\mathbf{7}}$ \\
\hline $\mathrm{A}$ & 7 & 4 & .4 & .1 & .1 & .1 & .1 & .1 & .1 & .4 & .2 & .2 & .2 & 0 & 0 & 0 \\
\hline B & 4 & 7 & .4 & .2 & .2 & .2 & 0 & 0 & 0 & .4 & .1 & .1 & .1 & .1 & .1 & .1 \\
\hline
\end{tabular}

Table 2. Results obtained for Model (A): $M>N$ case

\begin{tabular}{|l|l|l|l|}
\hline Model A & $\mathbf{d}=\mathbf{6}$ & $\mathbf{d}=\mathbf{7}$ & $\mathbf{d}=\mathbf{8}$ \\
\hline $\mathrm{P}(\mathrm{S}=0)$ & 0.475403191 & 0.511590931 & 0.544695509 \\
\hline $\mathrm{P}(\mathrm{S}=1)$ & 0.087658829 & 0.08766979 & 0.08692719 \\
\hline $\mathrm{P}(\mathrm{S}=2)$ & 0.053921363 & 0.053522714 & 0.052586088 \\
\hline $\mathrm{P}(\mathrm{S}=3)$ & 0.051042964 & 0.05030335 & 0.049085536 \\
\hline$\pi 0 \mathrm{e}$ & 0.80877548 & 0.838243532 & 0.862193111 \\
\hline$\pi 1 \mathrm{e}$ & 0.142711618 & 0.126142751 & 0.111336255 \\
\hline$\pi 2 \mathrm{e}$ & 0.035921733 & 0.027563082 & 0.021229286 \\
\hline $\mathrm{P}(\mathrm{S}>20)$ & 0.012591168 & 0.008050635 & 0.005241348 \\
\hline $\mathrm{E}(\mathrm{S})$ & 3.261592244 & 2.809576922 & 2.451698198 \\
\hline Var(S) & 24.47523225 & 19.75415308 & 16.32180546 \\
\hline Arrival rate & 1.328571429 & 1.269386292 & 1.27609882 \\
\hline Sales rate & 1.885714286 & 2.542161256 & 2.866098546 \\
\hline Norm & $3.70 \mathrm{E}-05$ & $3.08 \mathrm{E}-05$ & $2.25 \mathrm{E}-05$ \\
\hline & & & \\
\hline & & & \\
\hline & & & \\
\hline & & & \\
\hline
\end{tabular}

Table 3. Results obtained for Model (B): $M<N$ case

\begin{tabular}{|l|l|l|l|}
\hline Model B & $\mathbf{d}=\mathbf{6}$ & $\mathbf{d}=\mathbf{7}$ & $\mathbf{d}=\mathbf{8}$ \\
\hline $\mathrm{P}(\mathrm{S}=0)$ & 0.582798685 & 0.618645917 & 0.649239331 \\
\hline $\mathrm{P}(\mathrm{S}=1)$ & 0.100664197 & 0.097800786 & 0.094490671 \\
\hline $\mathrm{P}(\mathrm{S}=2)$ & 0.073421762 & 0.070060588 & 0.06662544 \\
\hline $\mathrm{P}(\mathrm{S}=3)$ & 0.071195817 & 0.067078568 & 0.063110424 \\
\hline$\pi 0 \mathrm{e}$ & 0.946584837 & 0.95933118 & 0.968289888 \\
\hline$\pi 1 \mathrm{e}$ & 0.049575736 & 0.038390264 & 0.030295733 \\
\hline$\pi 2 \mathrm{e}$ & 0.003559797 & 0.002148859 & 0.00135011 \\
\hline $\mathrm{P}(\mathrm{S}>20)$ & 0.00027963 & 0.000129697 & $6.43 \mathrm{E}-05$ \\
\hline $\mathrm{E}(\mathrm{S})$ & 1.500573303 & 1.289995146 & 1.129194057 \\
\hline Var(S) & 6.273535959 & 5.115246008 & 4.292091123 \\
\hline Arrival rate & 0.864403711 & 0.087537426 & 0.884032997 \\
\hline Sales rate & 2.967732567 & 3.42594126 & 3.881022836 \\
\hline Norm & $1.93 \mathrm{E}-05$ & $4.90 \mathrm{E}-05$ & $3.01 \mathrm{E}-05$ \\
\hline & & & \\
\hline & & & \\
\hline
\end{tabular}



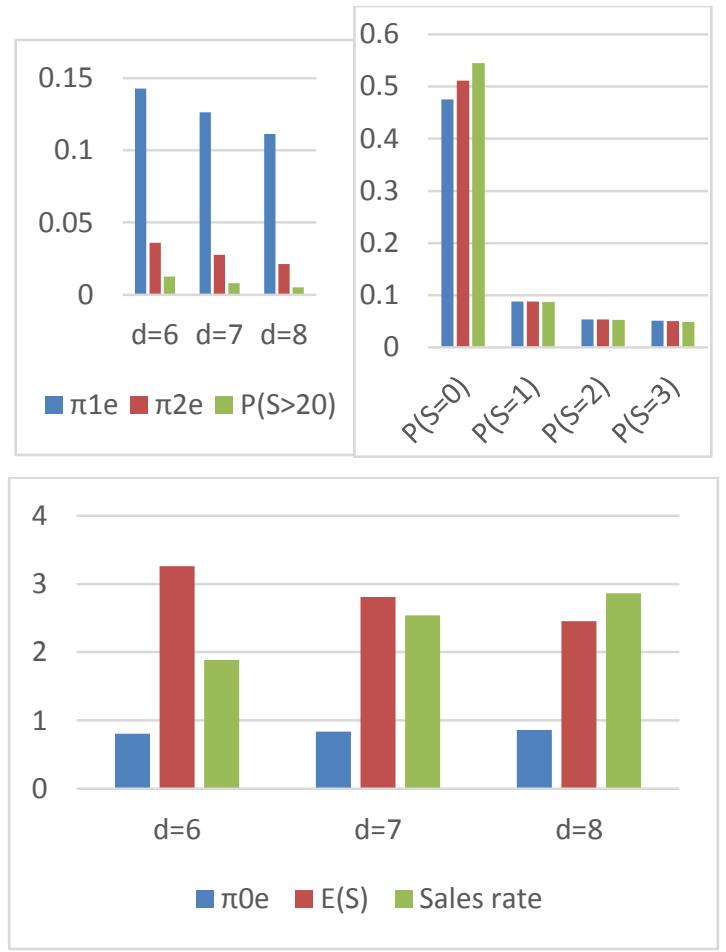

Figures 1, 2 and 3: Probabilities, E(S) and Sales rates for Model (A) Measures with $\mathrm{M}=7$ and $\mathrm{N}=3$.

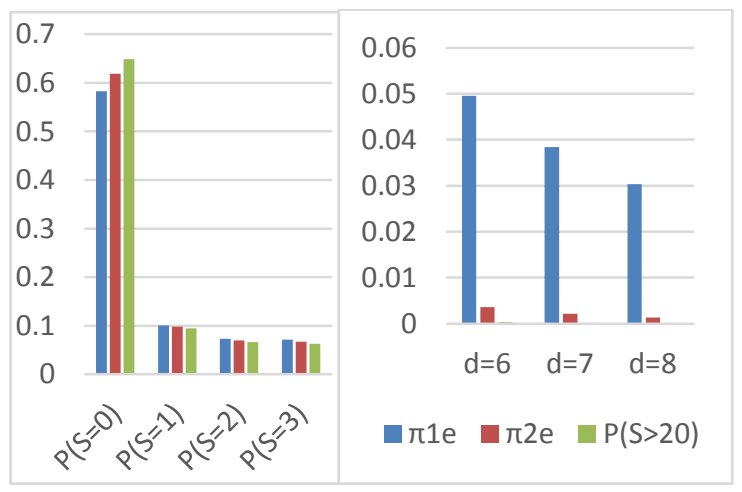

Figures 4, 5 and 6: Prob

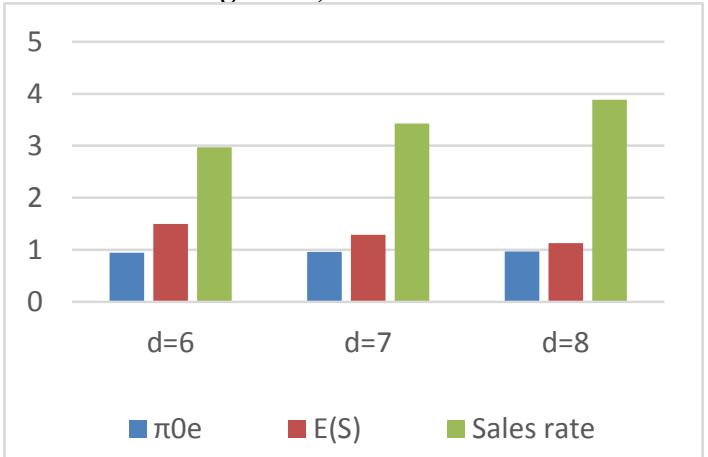

abilities, E(S) and Sales rates for Model (B) Measures with $\mathrm{M}=\mathbf{3}$ and $\mathrm{N}=7$.

\section{CONCLUSION}

Considering a continuous time Markov chain, Model (A) where the maximum production size is greater than the maximum sales size and its dual Model (B) are studied with catastrophic sales. The stationary probabilities, expected value, variance and probabilities of empty level are derived using the rate matrix iterated. Bulk arrival and bulk service queue becomes a special case of the model studied. When the catastrophic parameter becomes zero the model reduces to a bulk arrival and bulk service queue with both arrival and service batches of random sizes.. Numerical examples are presented for illustration and all performance measures including expected length, variance, and state probabilities are obtained. These models with random environment may be considered as a suitable and relevant new area for extension.

\section{REFERENCES}

[1] Aissani.A. and Artalejo.J.R. 1998. On the single server retrial queue subject to break downs, Queueing System $.30,309-321$.

[2] Ayyappan.G, Muthu Ganapathy Subramanian. A and Gopal Sekar. 2010. M/M/1 retrial queueing system with loss and feedback under pre-emptive priority service, IJCA, Vol. 2, N0.6, 27-34.

[3] D. Bini, G. Latouche, and B. Meini. Numerical Methods for Structured Markov Chains. Numerical Mathematics and Scientific Computation. Oxford University Press, Oxford, 2005. 327 pages.

[4] Chakravarthy.S.R and Neuts. M.F.2014. Analysis of a multiserver queueing model with MAP arrivals of special customers, Simulation Modelling Practice and Theory, vol.43, 79-95,

[5] Gaver, D., Jacobs, P., Latouche, G, 1984. Finite birthand-death models in randomly changing environments. Advances in Applied Probability 16, 715-731

[6] Latouche.G, and Ramaswami .V, (1998). Introduction to Matrix Analytic Methods in Stochastic Modeling, SIAM. Philadelphia, PA.

[7] Neuts .M.F.1981. Matrix- Geometric Solutions in Stochastic Models: An algorithmic Approach, The Johns Hopkins Press, Baltimore

[8] Neuts. M.F and Nadarajan.R, 1982. A multi-server queue with thresholds for the acceptance of customers into service, Operations Research, Vol.30, No. 5, 948-960.

[9] Noam Paz, and Uri Yechali, 2014 An M/M/1 queue in random environment with disaster, Asia- Pasific Journal of Operational Research 01/2014;31(30.DOI: 101142/S021759591450016X

[10] William J. Stewart, The matrix geometric/analytic methods for structured Markov Chains, www.sti.uniurb/events/sfmo7pe/slides/Stewart2pdfN.C.State University 